\title{
Brain Tumor Detection Using Histogram Thresholding to Get the Threshold point
}

\author{
${ }^{1}$ Easha Noureen, ${ }^{2}$ Dr. Md. Kamrul Hassan \\ Dept. of Electrical \& Electronic Engineering, American International University-Bangladesh \\ House 58/B, Road 4, Block B, Dhaka 1213, Bangladesh \\ e-mail: noureeneasha@gmail.com \\ Assistant Professor Faculty of Engineering American International University-Bangladesh \\ House 58/B, Road 4, Block B, Dhaka 1213, Bangladesh \\ e-mail:mdkamrul@aiub.edu
}

\begin{abstract}
In this paper, a computer-based method for defining tumor region in the brain using MRI images is presented. Today's modern medical imaging research faces the challenge of detecting brain tumor through Magnetic Resonance Images (MRI). Normally, to produce images of soft tissue of human body, MRI images are used by experts. It is used for analysis of human organs to replace surgery. For brain tumor detection, image segmentation is required. For this purpose, the brain is partitioned into two distinct regions. This is considered to be one of the most important but difficult part of the process of detecting brain tumor. Hence, it is highly necessary that segmentation of the MRI images must be done accurately before asking the computer to do the exact diagnosis. Brain tumor, a mass of tissue that grows out of control is one of the major causes for the increase in mortality among children and adults. Segmenting the regions of brain is the major challenge in tumor detection. A large number of effective segmentation algorithms have been used for segmentation in grey scale images ranging from simple edge-based methods to composite high-level approaches using modern and advanced pattern recognition approaches. Brain tumor detection is one of the challenging tasks in medical image processing. Four MRI images were collected and the experiments were conducted for statistical analysis also. A comparative study is made and the results are of great interest and practical utility.
\end{abstract}

Keywords: Brain tumor, Segmentation, Magnetic Resonance Imaging, Thresholding, Histogram

\section{Introduction}

Brain tumor, which is one of the most common brain diseases, has affected and devastated many lives. Statistics still shows low survival rate of brain tumor patients. To combat this, recently, researchers are using multi-disciplinary approach involving knowledge in medicine, mathematics and computer science to better understand the disease and find more effective treatment methods. In medical image processing brain tumor detection is one of the challenging tasks, since brain images are complicated and tumors can be analyzed only by expert physicians. So in this paper brain tumor detection is discussed by various methods. In this paper, the histogram is calculated and the threshold value is obtained and fixed. The analysis is carried out by using MRI image.

Magnetic resonance (MR) imaging and computer tomography (CT) scanning of the brain are the two most common tests undertaken to confirm the presence of brain tumor and to identify its location for selected specialist treatment options. Currently, there are different treatment options available for brain tumor. These options include surgery, radiation therapy, and chemotherapy. The choice for the treatment options depends on the size, type, and grade of the tumor. It also dependents on whether or not the tumor is putting pressure on vital parts of the brain. Whether the tumor has spread to other parts of the central nervous system (CNS) or body, and possible side effects on the patient concerning treatment preferences and overall health [2] are important considerations when deciding the treatment options.

MRI is the most widely used technique in the field of radio imaging.[1,2]. MR is a dynamic and flexible technology that allows acquisition of variable image contrast by using different pulse sequences and by changing the imaging parameters corresponding to longitudinal relaxation time (T1), and transverse relaxation time (T2). Signal intensities on T1 and T2 weighted images relate to specific tissue characteristics. The contrast on MR image is a factor dependent on pulse sequence parameters. The most common pulse sequences are T1weighted and T2-weighted spin-echo sequences. MR imaging of the body is performed to get the structural details of brain, liver, chest and pelvis which helps in diagnosis or monitoring the treatment.

Histogram thresholding is used by the Object Extraction, Background and Image Factory (segmentation) tools to select a range of pixel values that should match to what you want to select in the image. The histogram represents the intensity values of the visible part of the image only. Thresholding is a technique for converting a grayscale or color image to a binary image based upon two threshold values. The 
histogram presents the frequency of intensity values of a channel in an image .The threshold values (min and max) can be applied by moving the arrows above the histogram or by using an automatic threshold method..

This paper presents a review of the methods and techniques used during brain tumor detection through MRI image segmentation. The paper concludes with a discussion on the future trends of more advanced research on brain segmentation.

\subsection{Main parts of brain:}

\section{Brain Anatomy Overview}

The human brain which functions as the center for the control of all the parts of human body is a highly specialized organ that allows a human being to adapt and endure varying environmental conditions. The human brain enables a human to articulate words, execute actions, and share thoughts and feelings. In this section the tissue structure and anatomical parts of the brain are described to understand the purpose of this study. The brain is composed of two tissue types, namely gray matter (GM) and white matter (WM).Gray matter is made of neuronal and glial cells, also known as neuroglia or glia that controls brain activity and the basal nuclei which are the gray matter nuclei located deep within the white matter. The basal nuclei include: caudate nucleus, putamen, pallidum and claustrum. White matter fibers consist of many elinated axons which connect the cerebral cortex with other brain regions. The left and the right hemispheres of the brain are connected by corpus callosum which is a thick band of white matter fibers. The brain also contains cerebrospinal fluid (CSF) which consists of glucose, salts, enzymes, and white blood cells. This fluid circulates through channels (ventricles) around the brain and the spinal cord to protect them from injury. There is also another tissue called meninges which are the membrane covering the brain and spinal cord connected with the conscious thoughts, movement and sensations. It further consists of two halves, the right and the left hemispheres. Each controls the opposite side of the body. Moreover, each hemisphere is divided into four lobes: the frontal, temporal, parietal and occipital lobes. The cerebellum is the second largest structure of brain. It is connected with controlling motor functions of body such as walking, balance, posture and the general motor coordination. It is situated toward the back side of the brain and is linked to brain stems. Both cerebellum and cerebrum have a very thin outer cortex of gray matter, internal white matter and small but deeply situated masses of the gray matter. The spinal cord is connected to the brainstem. It is located toward the bottom of the brain. Brainstem controls vital functions in human body such as motor, sensory pathways, cardiac, repository and reflexes. It has three structures: the midbrain, pons and medulla oblongata .See the parts of the brain to learn what they do. Knowing the location of tumor(s) may help us to understand changes in how patient act or think. Changes can be due to the impact of the tumor itself or from treatment. For example, if patient has a tumor in the temporal lobe, you may have shortterm memory loss. Tumors can also be associated with difficulty with multi-tasking, seizures, and headaches.

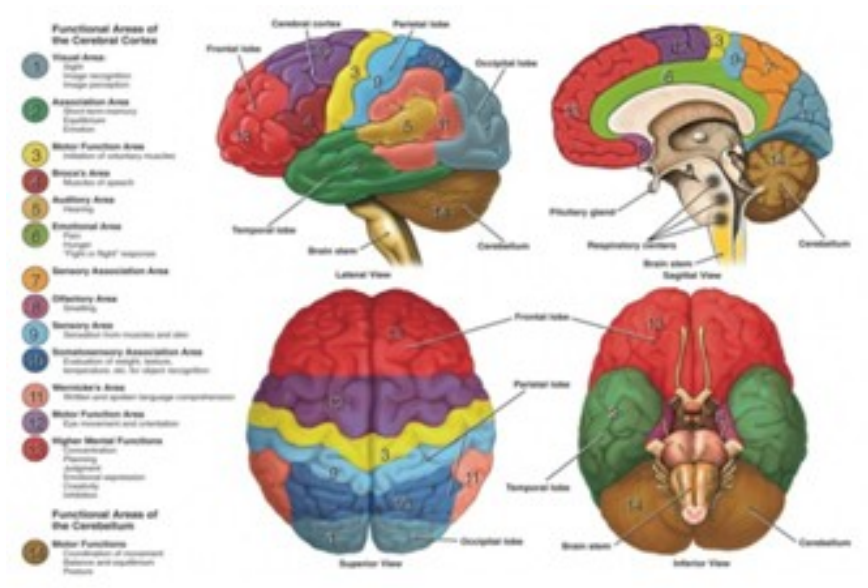

Figure1: Main parts of Brain

\subsection{Tumor:}

The word tumor is a synonym for a word neoplasm which is formed by an abnormal growth of cells Tumor is something totally different from cancer.

\section{1) Types of Tumor:}

There are three common types of tumor: 1)Benign ; 2) Pre-Malignant;3)Malignant(cancer can only be malignant). 
a) Benign Tumor: A benign tumor is a tumor is the one that does not expand in an abrupt way; it doesn't affect its neighboring healthy tissues and also does not expand to non-adjacent tissues. Moles are the common example of benign tumors.

b) Pre-Malignant Tumor: Premalignant Tumor is a precancerous stage, considered as a disease, if not properly treated it may lead to cancer.

c) Malignant Tumor: Malignancy (mal- = "bad" and -ignis = "fire") is the type of tumor, that grows worse with the passage of time and ultimately results in the death of a person. Malignant is basically a medical term that describes a severe progressing disease. Malignant tumor is a term which is typically used for the description of cancer.

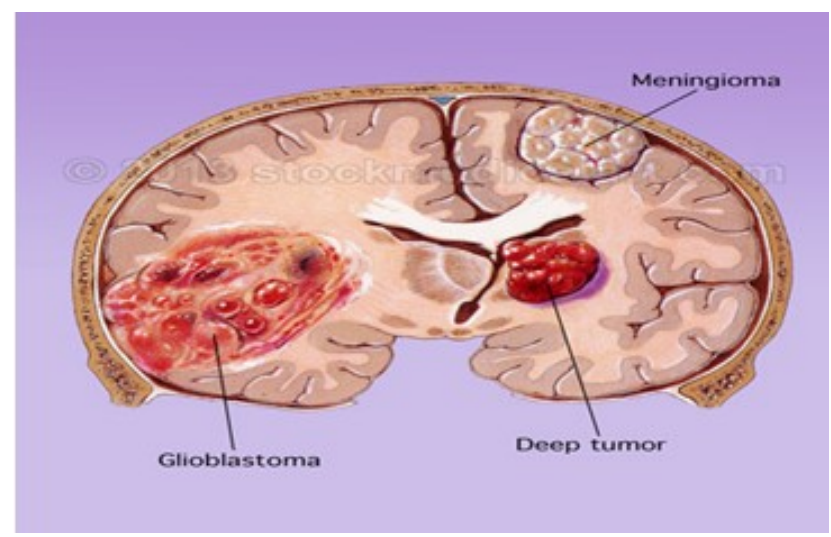

Figure2: Brain Tumor

\subsection{Magnetic Resonance Imaging (MRI):}

MRI is basically used in the biomedical to detect and visualize finer details in the internal structure of the body. This technique is basically used to detect the differences in the tissues which have a far better technique as compared to computed tomography. So this makes this technique a very special one for the brain tumor detection and cancer imaging.

CT uses ionizing radiation but MRI uses strong magnetic field to align the nuclear magnetization then radio frequencies changes the alignment of the magnetization which can be detected by the scanner. That signal can be further processed to create the extra information of the body.

\subsection{Histogram thresholding :}

\section{Amplitude segmentation based on histogram features:}

This includes segmentation of an image based on thresholding of histogram features and gray level thresholding and perhaps the simplest technique. This is particularly suitable for an image with region or object of uniform brightness placed against a background of different gray level. A threshold can be applied to segment the object and background. Threshold is defined mathematically as shown below:

$$
\mathrm{C}(\mathrm{i}, \mathrm{j})=\left\{\begin{array}{c}
255 p(i, j) \geq T \\
0 p(i, j) \leq T
\end{array} \ldots . .(1)\right.
$$

Where $c(i, j)$ is the resulting pixel at co-ordinate $(i, j) ; p(i, j)$ is the pixel of the input image and $T$ is the threshold value.

Equation 1 gives excellent results for segmentation of image. Thresholding operation, defined by equation-1 is very basic and simple, and works well only when the object and background have uniform brightness of distinct gray level values respectively. This threshold operation does not work well at segmentation of images with multiple objects each having distinct gray level value varying over a band of values. To overcome this limitation, band thresholding based multiple thresholding operation is applied:

$$
\mathrm{C}(\mathrm{i}, \mathrm{j})=\left\{\begin{array}{l}
1 \text { for } T 1<p(i, j) \leq T 2 \\
2 \text { for } T 2<p(i, j) \leq T 3 \ldots \ldots(2) \\
k \text { for } T k<p(i, j) \leq T k
\end{array}\right.
$$

0 otherwise.

Here, the kth band corresponds to the object or region having pixel values in the range of Tk to Tk-1 where $\mathrm{Tk}$ is the lower limit of gray level and $\mathrm{Tk}+1$ is the upper limit of gray level band. 
For application of thresholding based segmentation technique, it is required to apply the correct threshold values in order to achieve proper segmentation results, otherwise results are poor.

\section{Algorithm for segmentation through histogram thresholding:}

Step 1: The MRI image of the brain is divided into two equal halves around its central axis and the histogram of each part drawn. This will detect the infectious side of the brain.

Step 2: The threshold point of the histograms is calculated based on a comparison technique made among the two histograms.

Step 3: Segmentation is done using the threshold point for both the halves.

Step 4: The detected image is cropped along its contour to find out the physical dimension of the tumor.

Step 5: Create an image of the original size, check the segmented images pixel value; if it's value is greater than threshold value, assign 255 else 0 .

Step 6: Segmented image is displayed.

Step 7: Find the physical dimension of the tumor, using the following algorithm.

(i) Total number of the pixels, having pixel value 255 is found using the following command.

total=bwarea(segmented image)

(ii) Resolution of $\mathrm{X}$ and $\mathrm{Y}$ axis is found.( Resolution is obtained from image information)

$\mathrm{a}=1 /$ xresolution* $1 /$ yresolution

(iii) Area of the tumor is found by the following statement.

area of the tumor=total*a

\section{Experiments and Results}

In this section the results of the experiments conducted on the data set (image) are presented and discussed. Codes are written and using MATLAB the desired results are obtained.

\section{Plot the histogram}

Histogram is a plot between number of pixel and pixel intensity. To plot the histogram, bar graph can be used. The histogram code operates by first reading the grayscale value at the first entry and coming up with pixel intensity between 0 and 255. It increments the total number of pixels and then it will move on to the next row or column entry until it finishes reading all the raster data. However, while it is reading each entry, if it picks up pixel intensity value more than once it will increment that particular value.

\section{Choose threshold point}

The difference of the two histograms is calculated and the resultant difference is plotted using bar graph to select the threshold point.

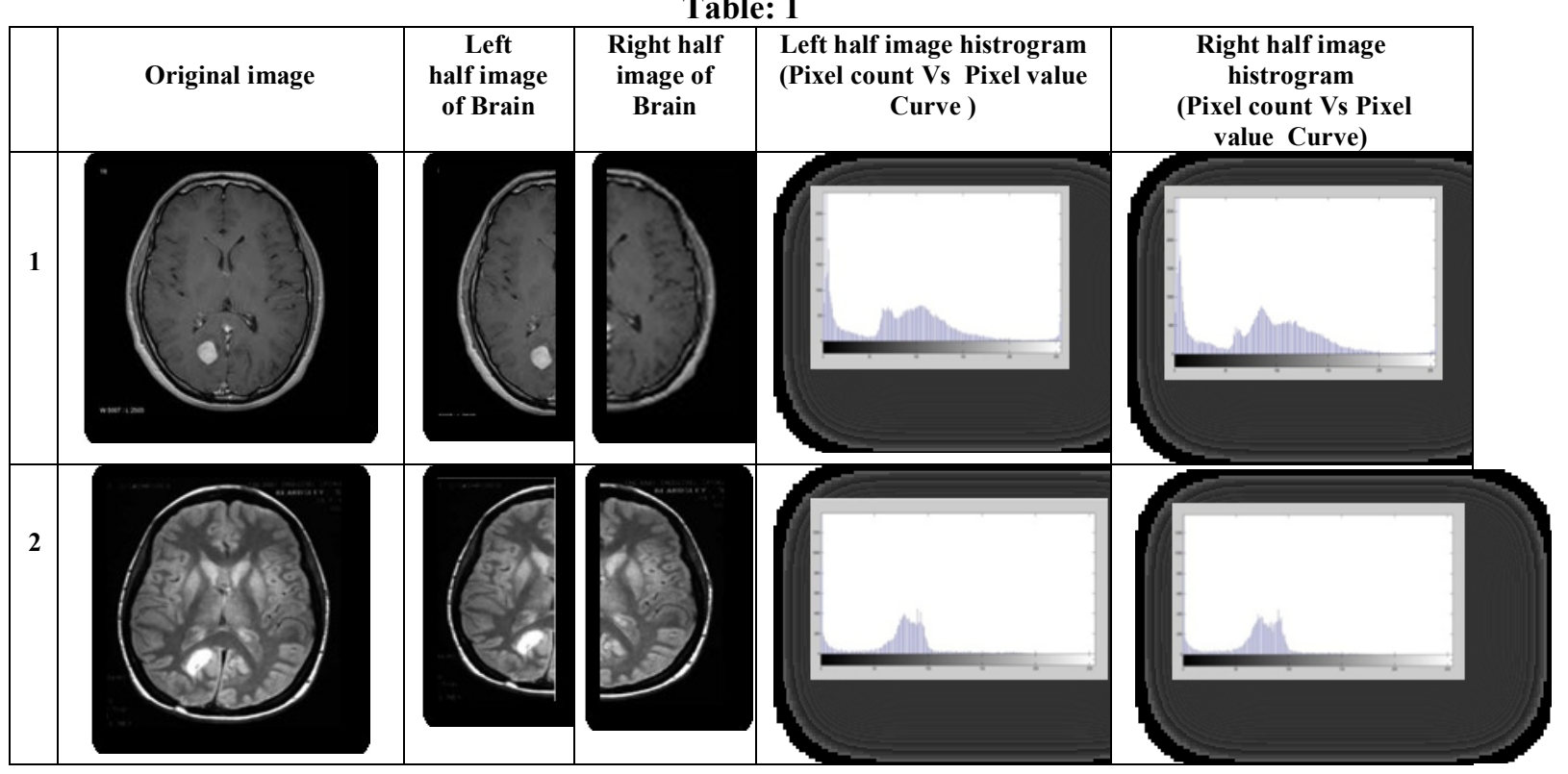



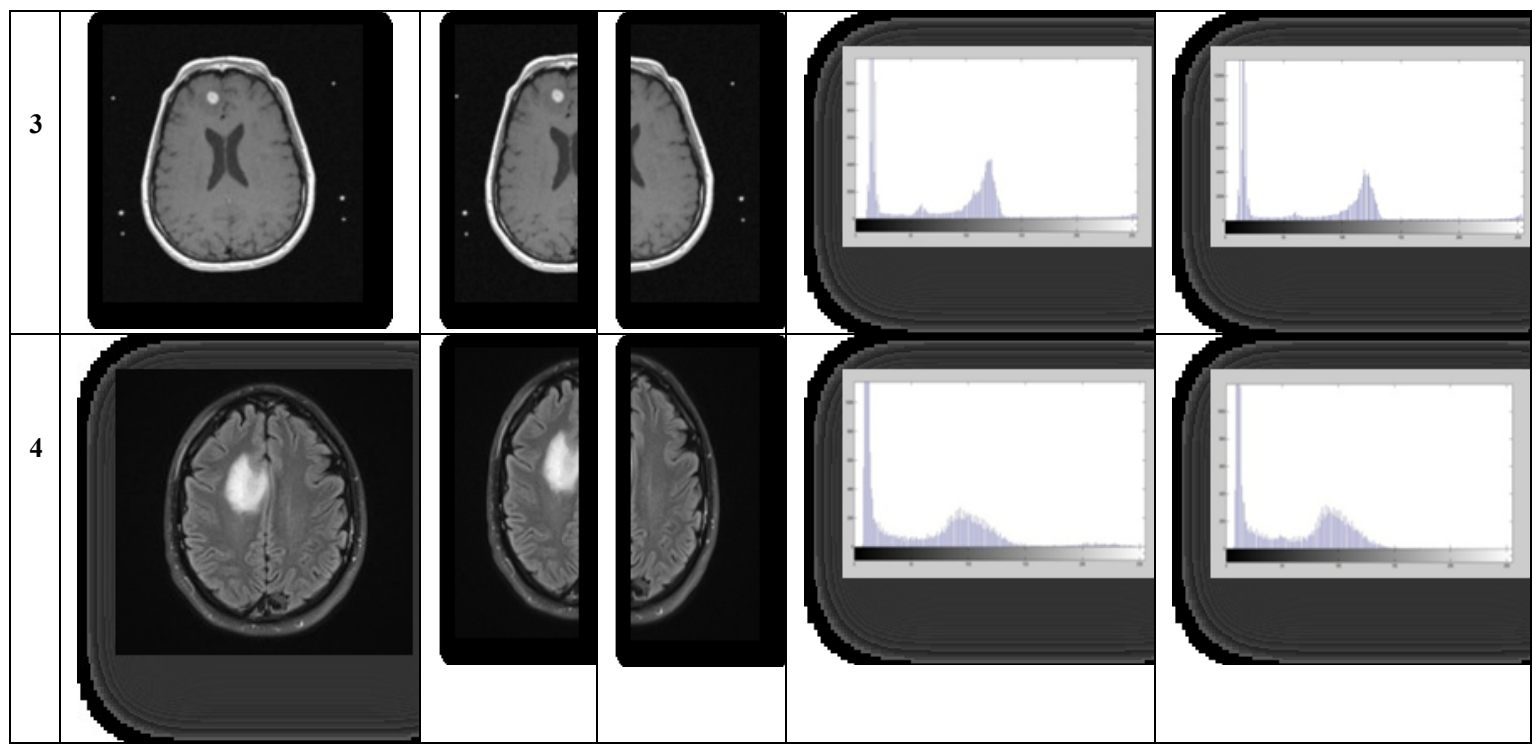

Table:2

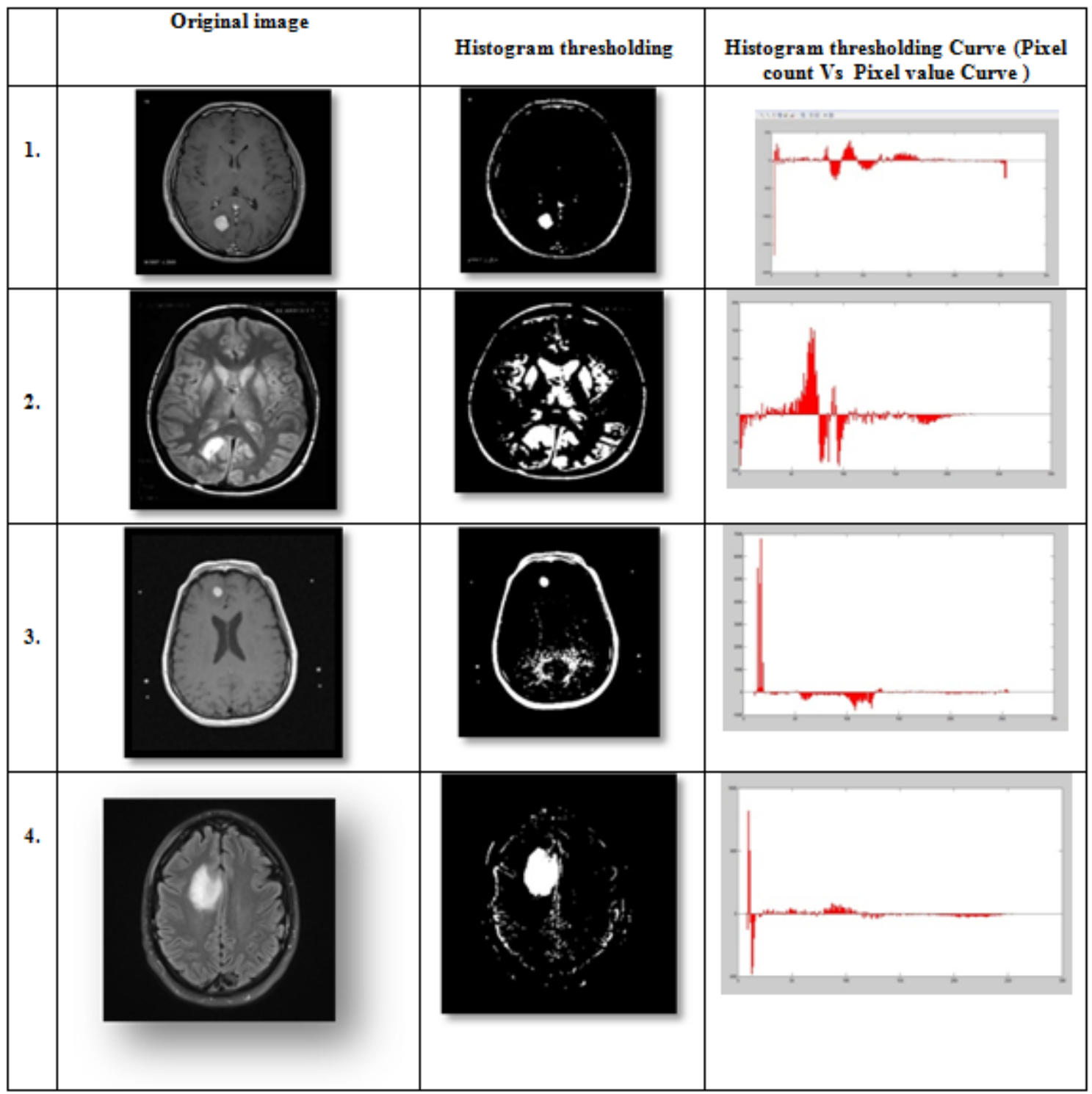




\section{Conclusion}

Computer-aided segmentation is a key step for finding application in computer aided diagnosis, clinical studies and treatment planning. In recent years a variety of approaches have been proposed to segment MR and CT images, which has its own merits and limitations. This study provides the results of different segmentation approaches and their respective statistical analysis.

The algorithms were applied on four sample images and the results obtained were found to be extremely good and efficient. The proposed algorithm can be applied with certain modification for detection of lungs cancer. In all the cases codes are written and implemented meticulously. Finally, it is concluded that the results of the present study are of great importance in the brain tumor detection which is one of the challenging tasks in medical image processing.

\section{References}

[1]. Prince JL, Links JM. Medical imaging signals and system. Pearson Education. 2006

[2]. Macovski A. Medical imaging systems. Prentice; 1983

[3]. Pratibha Sharma,Sangam Choudhary and Manoj Diwakar, Dept.of Computer Science, MITS, Lakshmangarh, "Application of Edge Detection for Brain Tumour Detection", International Journal of Computer Applications (0975 - 8887), Volume 58- No.16, November 2012-21.

[4]. Yehualashet Megersa, Electrical and Computer Engineering Department, Addis Ababa University, "Brain Tumour Detection and segmentation using Hybrid Intelligent Algorithm", November 2012.

[5]. Sezgin M. and Sankur B., "Survey over image thresholding techniques and quantitative performance evaluation", Journal of electronic Imaging, Vol. 13, No. 1, Jan. 2004, pp 146-165

[6]. S.Xavierarockiaraj, K.Nithya and R.Maruni Devi, "Brain tumour Detection Using Modified Histogram thresholding-Quadrant Approach", Hournal of Computer Applications(JCA), Vol. 5, No.1, 2012, pp.21-25.

[7]. Anam Mustaqeem, ali Javed and Tehseen Fatima, "An Efficient Brai tumour Detection Algorithm using Watershed and Thresholding based segmentation", International Journal of Image, Graphicss and signal Processing (IJIGSP), Vol. 4, No.10, 2012, pp.34-39.

[8]. Kai Xie, Jie Yang, Z.G.Zhang and Y.M.Zhu, "Semi-sutomated brain tumour and edema segmentation using MRI:, European Journal of Radiology, Vol.56, Issue 1,October 2005, pp.12-19

[9]. Nikola K. Kasabov, Foundations of Neural Networks, Fuzzy systems and Knowledge Engineering, Massachusetts Institute of Technology, 1998, pp.167-473

[10]. M.Egmont-Petersen, D. de Ridder and H.Handels, "Image Processing with Neural Networks- a review", Pattern Recognition, Vol.35, Issue 10 Oct. 2002, PP. 2279-2301

[11]. Vishal B.Padole, D.S.Chaudhari, "Detection of brain tumour in MRI Images using mean shift algorithm and normalized cut method" Department of Electronics and Telecommunication, Government College of Engineering, Amravati Maharashtra, India. International Journal of Engineering and Advanced Technology (IJEAT), ISSN:2249-8958, volume-1, Issue-5, June 2012

[12]. Dina Aboul Dahab, Samy S.A.Ghoniemy, Gamal M.Selim, Dept. of Computer Engineering, Arab Academy for Science , Technology and Maritime Transport Cairo, Egypt. "Automated Brain Tumour Detection and Identification using Image processing and probabilistic neural network techniques". International Journal of Image Processing and Visual communication ISSN 23191723: Volume 1, Issue 2, October 2012.

[13]. T. Logeswari, Mother Teresa Women's college. and M. Karnan, College of Engineering, Coimbore. Anna University, Tamil Nadu, "An improved implementation of brain tumour detection using segmentation based on soft computing", Journal of Cancer Research and Experimental Oncology, JCREO, vol. 2(1) pp. 006-014, March, 2010.

[14]. Abhishek Raj, Alankrita, Akansha Srivastava and Vikrant Bhateja, "Computer Aided Detection of Brain Tumour in Magnetic Resonance Images", IACSIT, Internal Journal of Engineering and Technology, Vol. 3, No. 5, October 2011.

[15]. Anam Mustaqeen, Ali Javed and Tehseen Fatima, Department of Software Engineering, UET Taxila, “An Efficient Brain Tumour Detection Algorithm Using Watershed \& Thresholding Based Segmentation”, I.J.Image, Graphics and Signal Processing, 2012, 10, 34-39

[16]. Li H, Deklerck R, De Cuyper B, Hemanus A, Nyssen E, Comelis J, "Object recognition in brain CT-Scan:Knowledge-based fusion of data from multiple feature extractors". IEEE Trns Med Imaging. 2005; 14(2):12-29

[17]. Pham DL, prince JL, Dagher AP, Xu C. "An automated technique for statistical characterization of brain tissues in magnetic resonance imaging. Int J.Patt rec Art Intel 2007;11:1189-211

[18]. Gonzalez RC, Woods RE. Digital image processing. 2nd ed. 2004. Pearson Education.

[19]. Pratt KW. Digital image processing. 3rd ed. Willey; 2001. PP.551-87.

[20]. Pal NR, Pal SH. "A review on image segmentation technique”. Pattern Recog. 1993; 26:1277-97.

[21]. P.K.Srimani and Shanthi Mahesh, "Knowledge Discovery in Image Segmentation data using Decision Tree classifiers", IJCR, vol.4, issue, 09, pp.135-140, September, 2012.

[22]. P.K.Srimani and Shanthi Mahesh, "Knowledge Discovery process in the Image-segmentation Data", IJKE, Vol 3, Issue 2, 2012 , pp. 188-192 